Western University

Scholarship@Western

$1-1-2012$

\title{
Modeling stochastic and spatial heterogeneity in a human airway tree to determine variation in respiratory system resistance
}

Del Leary

Swati A Bhatawadekar

Grace Parraga

Geoffrey N Maksym

Follow this and additional works at: https://ir.lib.uwo.ca/biophysicspub

Part of the Medical Biophysics Commons

\section{Citation of this paper:}

Leary, Del; Bhatawadekar, Swati A; Parraga, Grace; and Maksym, Geoffrey N, "Modeling stochastic and spatial heterogeneity in a human airway tree to determine variation in respiratory system resistance" (2012). Medical Biophysics Publications. 116.

https://ir.lib.uwo.ca/biophysicspub/116 


\title{
Modeling stochastic and spatial heterogeneity in a human airway tree to determine variation in respiratory system resistance
}

\author{
Del Leary, ${ }^{1}$ Swati A. Bhatawadekar, ${ }^{2}$ Grace Parraga, ${ }^{3,4,5}$ and Geoffrey N. Maksym ${ }^{1,2}$ \\ ${ }^{1}$ Department of Physics and ${ }^{2}$ School of Biomedical Engineering, Dalhousie University, Halifax; ${ }^{3}$ Imaging Research \\ Laboratories, Robarts Research Institute, London; and ${ }^{4}$ Department of Medical Biophysics and ${ }^{5}$ Graduate Program \\ in Biomedical Engineering, The University of Western Ontario, London, Canada
}

Submitted 25 May 2011; accepted in final form 6 October 2011

Leary D, Bhatawadekar SA, Parraga G, Maksym GN. Modeling stochastic and spatial heterogeneity in a human airway tree to determine variation in respiratory system resistance. $J$ Appl Physiol 112: 167-175, 2012. First published October 13, 2011; doi:10.1152/japplphysiol.00633.2011.-Asthma is a variable disease with changes in symptoms and airway function over many time scales. Airway resistance (Raw) is variable and thought to reflect changes in airway smooth muscle activity, but just how variation throughout the airway tree and the influence of gas distribution abnormalities affect Raw is unclear. We used a multibranch airway lung model to evaluate variation in airway diameter size, the role of coherent regional variation, and the role of gas distribution abnormalities on mean Raw ( $\bar{R} a w)$ and variation in Raw as described by the SD (SDRaw). We modified an anatomically correct airway tree, provided by Merryn Tawhai (The University of Auckland, New Zealand), consisting of nearly 4,000 airways, to produce temporal and spatial heterogeneity. As expected, we found that increasing the diameter variation by twofold, with no change in the mean diameter, increased SDRaw more than fourfold. Perhaps surprisingly, $\bar{R} a w$ was proportional to SDRaw under several conditions-when either mean diameter was fixed, and its SD varied or when mean diameter varied, and $\mathrm{SD}$ was fixed. Increasing the size of a regional absence in gas distribution (ventilation defect) also led to a proportionate increase in both $\bar{R} a w$ and SDRaw. However, introducing regional dependence of connected airways strongly increased SDRaw by as much as sixfold, with little change in $\bar{R} a w$. The model was able to predict previously reported Raw distributions and correlation of SDRaw on Raw in healthy and asthmatic subjects. The ratio of SDRaw to $\bar{R} a w$ depended most strongly on interairway coherent variation and only had a slight dependence on ventilation defect size. These findings may explain the linear correlation between variation and mean values of Raw but also suggest that regional alterations in gas distribution and local coordination in ventilation amplify any underlying variation in airway diameters throughout the airway tree.

lung impedance; asthma; ventilation defects; variability

ASTHMA IS A VARIABLE DISEASE, with symptoms that are intermittent and variable in magnitude (25). Changes in airway bronchoconstriction are cardinal features of asthma, varying over multiple time scales from months to minutes (10). Most commonly, variation in airway function has been evaluated using changes in peak expired flow or variation in airway resistance $(R a w)$, both of which have been associated with airway diameter alterations $(2,13)$.

On the time scale of minutes, variation in respiratory system resistance (Rrs) can be monitored using the forced oscillation technique (FOT), and it is consistently reported that Rrs varies

Address for reprint requests and other correspondence: G. N. Maksym, School of Biomedical Engineering, Dalhousie Univ., 5981 Univ. Ave., P.O. Box 15000, Halifax, Canada B3H 4R2 (e-mail: gmaksym@dal.ca). continuously $(9,22)$. The magnitude of the variation is related to changes that affect airway diameter and are thought to be affected by the activity of the airway smooth muscle. Indeed, bronchoconstriction via methacholine increases the SD in Rrs $(\operatorname{SDR} r s)(9,22)$, and bronchodilation via albuterol decreases SDRrs (17). In a similar fashion, unloading and narrowing of the airways in the supine position increase SDRrs (22). The fluctuations in Rrs are also higher in asthma in both children and adults $(8,9,17,22)$. However, SDRrs is also highly correlated with Rrs, indicating that like Rrs, SDRrs depends on airway diameter; but, why these may be so tightly coupled is not well understood (9). Moreover, it not established how heterogeneity of ventilation, recognized to occur in asthma, would affect Rrs, measured at the airway opening $(6,24)$.

Animal studies suggest that airway obstruction is heterogeneous (3), modeling studies predict heterogeneous airway narrowing (31), and imaging of ventilation in asthma has also identified marked spatial heterogeneity in regional lung ventilation $(14,24)$. Furthermore, heterogeneity in ventilation from hyperpolarized helium-3 $\left({ }^{3} \mathrm{He}\right)$ imaging is quantitatively higher in asthma (30) and has been associated with changes in lung mechanics by forced oscillation in dogs (14) and in normal and asthmatic subjects $(4,28)$. These studies imply dramatic differences in regional airway narrowing, which occur within the lung. It has been argued that in airway obstruction, many airways may be changing diameter together continuously, both randomly and by changes in activation and airway loading, which leads to the reported continuous variation in Rrs, measured by forced oscillation (22). However, it is yet unclear how alterations in airway diameter in a multibranch lung can lead to the observed variation of Rrs.

Here, we examined how stochastic changes in airway diameter within a geometrically accurate airway tree may explain the statistical distribution of Rrs, described via Rrs and SDRrs. We developed a multibranch airway model to characterize how statistical variation in airway diameters could alter both the mean Raw ( $\bar{R} a w)$ as well as its SD (SDRaw), exploring the effects of airway narrowing or mean airway diameter and altered variation amongst airway diameters via the SD of airway diameters. We also explored the effect of interaction amongst airway diameters, via correlated and uncorrelated airway diameter variation, and the effects of large-scale heterogeneity via the introduction of large ventilation abnormalities-or defects. Predictions of Raw and its SD were then compared with previously reported FOT data obtained from healthy and asthmatic subjects. 


\section{METHODS}

Multibranch airway tree. The lung model developed here was based on an anatomically correct, three-dimensional human airway geometry, as previously described and provided by Merryn Tawhai (The University of Auckland, New Zealand) (26), up to the 12th generation governing 3,915 airways. The airway tree model was generated by using a subject-specific host volume derived from X-ray multidetector computed tomography (CT) imaging (27). Beyond the eighth generation, a volume-filling algorithm (27) was used to generate the remaining airway tree, maintaining daughter diameter ratios consistent with established morphometry (12). The airway tree was terminated at the 12th generation, similar to Venegas et al. (31), which represented airway diameters $\sim 1 \mathrm{~mm}$ in size. This end point was chosen as a compromise between computational feasibility and realistic impedance predictions. Since the Tawhai model is at total lung capactiy (TLC), we reduced the airway diameters to 0.7 of the Tawhai values, which assumed a homogenous volume change with a ratio of functional residual capacity (FRC) to TLC of 0.3 . This serves as a reference point only, as airway diameters were altered over a wide range, as described below.

The impedance of the airway tree for a given realization of airway diameter distribution was calculated using a lumped element approach, where the impedance of the airways was summed following the branching patterns using well-known series and parallel network impedance relations. Raw for each branch was approximated using Pouseille's flow given by

$$
R=\frac{128 \eta L}{\pi d^{4}}
$$

where $\eta$ is the dynamic viscosity of humid air at $37^{\circ} \mathrm{C}, L$ is the length, and $d$ is the diameter of the airway.

Each terminal airway was prescribed an elastance $E$ to act functionally as the peripheral airway wall compliance distal to the 12th generation in our model, and alveoli were calculated from the product of the number of terminal airways and normal elastance of the lung (29). This neglects any contribution to the impedance from gas compression or central or intermediate airway wall compliance, but these are both much smaller than the contribution from the peripheral airways and alveoli. The impedance for a terminal airway was defined as

$$
Z=R+i I \omega-\frac{i E}{\omega}
$$

and airway impedance for a nonterminal airway was calculated as

$$
Z=R+i I \omega
$$

where $I$ is the inertance calculated as $\rho_{\text {air }} L /\left(\pi r^{4}\right)$, with $\rho_{\text {air }}$ the air density, $L$ the length of a branch, and $r$ its radius; $E$ is the elastance; and $\omega$ is the angular FOT frequency. This resulted in a resonant frequency near $6 \mathrm{~Hz}$, which is normal in humans. The airway impedance of the entire airway tree could then be calculated numerically and separated further into real (resistance) and imaginary (reactance) parts. Because parallel pathways in a heterogeneous lung can lead to the product of reactances, the real part of the total impedance will have some dependence on the elastance and inertance, as was demonstrated originally in the two-compartment parallel Otis lung model (19).

Simulations. In a multibranch airway tree, the magnitude of variation of Raw at the airway opening is affected by the magnitude of variation at the level of the airway diameter and as will be demonstrated, the degree to which airways behave similarly and the presence of ventilation defects. Each simulation consisted of at least 2,000 repeated realizations of the model with each airway diameter varied, according to a prescribed distribution with a set mean and SD relative to the initial airway diameter from the Tawhai model, as described in more detail below. From each realization, we computed a Raw value, and from all of the realizations for a given simulation, we obtained a probability distribution for Raw. To explore the effect of the magnitude of variation in diameters, we first considered how increasing the amount of diameter variation throughout the lung would affect the total respiratory resistance. This would occur if airway smooth muscle activity contributed to increasing variability of airway diameters, as had been previously postulated, for example (22). While we did not include periodic contributions to variations in airway diameter, such as during lung volume changes associated with breathing, we also examined all simulations at different mean airway diameters, representing changes in average airway constriction or changes in lung volume, which would occur with breathing. Furthermore, we investigated how interactions between airways affected the distribution of Raw, which we describe as an incoherent or coherent variation, depending on whether parent- and daughter-connected airways vary independently or vary together. Finally, we investigated how different sizes of ventilation defects affected the distribution of Raw. Each of these simulations is described below.

Variation of the airway diameters. Variation in airway diameters throughout the airway tree model leads to total variation in resistance of the lung. The variation in airway diameters was controlled by setting a prescribed mean and SD relative to the initial diameters set by the initial airway geometry given in the Tawhai model.

$$
d_{i}=d_{\text {base }, i} \cdot N_{i}\left(\mu_{d}, \sigma_{d}\right)
$$

where $d_{i}$ is the diameter of a particular airway for a given realization of the model, $d_{\text {base }, i}$ is the diameter of a particular airway from the Tawhai model, and $N_{i}\left(\mu_{d}, \sigma_{d}\right)$ is a random number from a modified Gaussian distribution with relative airway diameter $\mu_{d}$ and relative airway diameter SD $\sigma_{d}$. For the different simulations, $\sigma_{d}$ was either held constant while airway diameter was changed (fixed variation model), altered at different fixed mean diameters (fixed mean model), or adjusted in proportion to the mean airway diameter (fixed slope; slope $_{d}$ model). Airway diameters were changed below their FRC baseline by altering the mean of the relative airway diameter distributions as

$$
\mu_{d}=\left(1-\frac{\xi}{100}\right)
$$

with $\xi=2.5,5,7.5,12.5,17.5,22.5$, and $27.5 \%$. Variation was changed by altering the SD of airway diameters $\sigma_{d}$ as

$$
\sigma_{d}=\lambda \mu_{d}
$$

with $\lambda=0.08,0.12,0.16$, and 0.2 .

The constant slope $_{d}$ model (Fig. 1A) was evaluated, as the results could be compared with the analytical solution in a single airway model by Lall et al. (17). An illustration of how the diameter distribution was altered for the fixed mean model and the fixed slope model is shown in Fig. $1 B$. As mentioned previously, this process was repeated for the entire airway tree for a minimum of 2,000 Raw calculations of different airway diameters, randomly chosen within the specified distribution to compute the statistical mean $(\bar{R} a w)$ and SD (SDRaw) of the entire model (Fig. 1D).

The diameters of all airways were randomly chosen from a modified Gaussian distribution to prevent extremely low values of airway diameter as follows: we limited the maximum narrowing for generations zero to four to be no less than $50 \%$ of their baseline diameter, representing the effect of cartilage-limiting airway narrowing, and any airway from generations five to 12 was limited to narrowing by $90 \%$, i.e. to $10 \%$ of its baseline diameter value. These limits were invoked infrequently, typically occurring $<1.2 \%$ of the time at $27.5 \%$ mean constriction, with SD equal to $10 \%$ of the mean diameter. Also, diameters were restricted from exceeding twice the constricted mean.

Airway diameter variation and regional coherency. To investigate the possibility that airways in a localized region may vary similarly, 


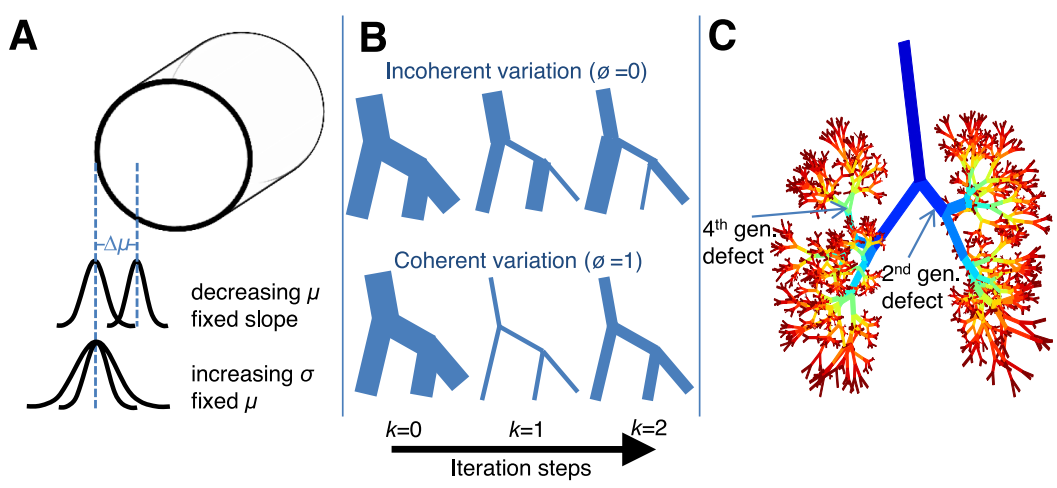

Fig. 1. A: the diameters of airways ( $\mu$ and $\sigma$ ) are varied at each iterative step by adjusting the diameter to a prescribed distribution, maintaining either a fixed mean, fixed SD (not shown), or fixed slope. $B$ : diameters of adjacent airways could vary at various levels of coherent variation $(\varphi)$. Shown here are examples of either fully incoherent or fully coherent variation between mother and daughter airways. $C$ : the multibranch airway tree consists of 12 generations for airway-resistance (Raw) predictions, with the colors here representing the airway generation number. The resistance $(D)$ for the entire airway tree is calculated at each iterative step.

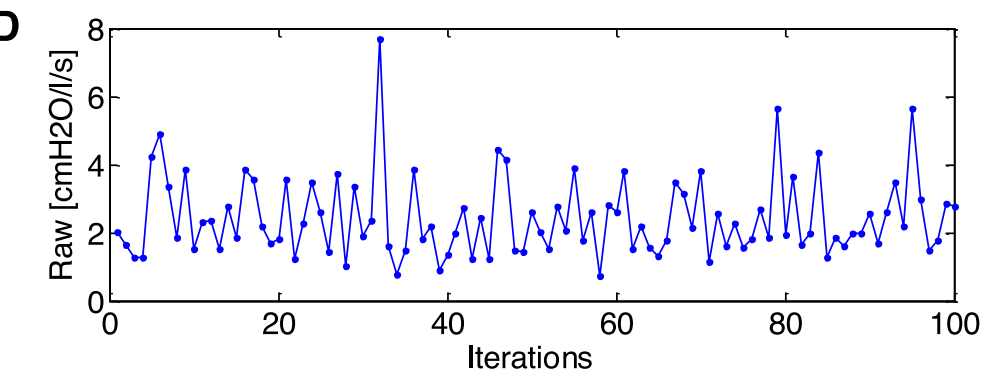

as might be a result of physical attachment or local similarity in activation or mechanical properties, we introduced a variable, $\varphi$, which could control the degree of spatial coherent variation in connected airways in a gradual and regional manner, thus generating regional heterogeneity. The diameter of the daughter airway, for example, $d_{i+1}$, was then determined either independently or in relation to the parent airway $d_{i}$, according to

$$
d_{i+1}=\varphi d_{i}\left(\frac{d_{\text {base }, i+1}}{d_{\text {base }, i}}\right)+(1-\varphi) d_{\text {base }, i+1} \cdot N_{i+1}\left(\mu_{d}, \sigma_{d}\right)
$$

The coherence parameter, $\varphi$, is bound between zero and one. For $\varphi=$ $0, d_{i+1}$ is independent from its parent airway and would vary with no coherent change in diameter, relative to its parent airway. For $\varphi=1$, $d_{i+1}$ is fully, coherently varying with its parent airway and thus all other airways throughout the lung. At $\varphi=0.6$, the parent is $60 \%$ coherent with its daughter and $36 \%$ with its granddaughter, with decreasing coherence for further generations distant to the parent. Thus larger values of $\varphi$ increase the degree of regional spatial coherency (Fig. 1B).

Simulating gas distribution abnormalities. Studies using hyperpolarized ${ }^{3} \mathrm{He}$ MRI have identified spatial and temporal heterogeneity in airway function. These are specific to obstructive lung diseases, such as asthma, or can be produced in healthy subjects with induced bronchoconstriction. Focal ventilation defects, where there is a ${ }^{3} \mathrm{He}$ signal void in anatomical regions related to airways narrowing, can be visualized and quantified $(7,23)$. Such ventilation defects can be significantly large compared with the volume of the lung and spatially and temporally persistent (6). To explore the effect of such apparent ventilation defects and more specifically, the airway narrowing, which we hypothesize is the determinant of such functional abnormalities, we modeled a single ventilation defect at different airway generations to explore the effect of defect size on $\bar{R} a w$ and SDRaw. In our model, we assumed that ventilation defects were generated by constricting a single airway at a given generation to $10 \%$ of its initial baseline value (Fig. 1C). While the etiology of ${ }^{3} \mathrm{He}$ MRI ventilation defects is yet unclear, we made the assumption that in asthma, defects are related to airway narrowing.

Single airway model. To help validate the multibranch model and to investigate the role of the transformation of the nonlinear transformation between airway diameter and Raw, we compared our predicted
$\bar{R} a w$ from the multibranch airway model with totally coherent variation with a single airway model with varying airway diameter distributions. The single airway radius was derived from the arithmetic mean of all of the airways in the multibranch lung model, and the length was then altered to achieve the same baseline resistance as the multibranch model.

Comparison with recorded distribution of airway impedance. We compared model-predicted distributions of $\bar{R} a w$ with Rrs, obtained from asthmatic and normal subjects, as reported previously (22). The study from which these data were obtained was approved by the Ethics Review Board of the Montreal Chest Research Institute, McGill University (Quebec, Canada), and subjects gave written, informed consent. The six healthy subjects (five men and one woman) had no history of pulmonary disease and were not hyper-reponsive by methacholine challenge, and asthmatic subjects were identified by the respirologist in charge of the clinic and had 1-s forced expiratory volume $\left(\mathrm{FEV}_{1}\right)$ measurements that varied between $64 \%$ and $92 \%$ predicted, taken prior to being tested. The FOT data from this study are from $6 \mathrm{~Hz}$ pressure oscillations-digitized at $256 \mathrm{~Hz}$ over $15 \mathrm{~min}$ of normal breathing, providing up to 5,400 values, excepting artifacts from coughs, swallows, or glottal closures, which were removed as described (22). The average of the mean resistances from the normal patients was 1.86 (SD 2.60) $\mathrm{cmH}_{2} \mathrm{O} \cdot 1^{-1} \cdot \mathrm{s}^{-1}$ and from the asthmatic patients was $5.20(\mathrm{SD} 3.35) \mathrm{cmH}_{2} \mathrm{O} \cdot 1^{-1} \cdot \mathrm{s}^{-1}$.

\section{RESULTS}

Increasing airway diameter. When the relative average airway diameter was changed, but the SDs of airway diameters kept constant, $\bar{R} a w$ increased as expected. However, the SDRaw also increased, as seen by the increase in the width of the distribution of Raw, despite no change in airway diameter variation (Fig. 2A).

Increasing airway diameter variation. Similarly, when the SD of airway diameters $\sigma_{d}$ was increased, while keeping the mean diameters $\mu_{d}$ fixed, SDRaw increased as expected; however, $\bar{R} a w$ also increased, despite no change in the $\mu_{d}$ (Fig. $2 B$ ). In both cases, $\bar{R}$ aw and SDRaw increased proportionately, but 

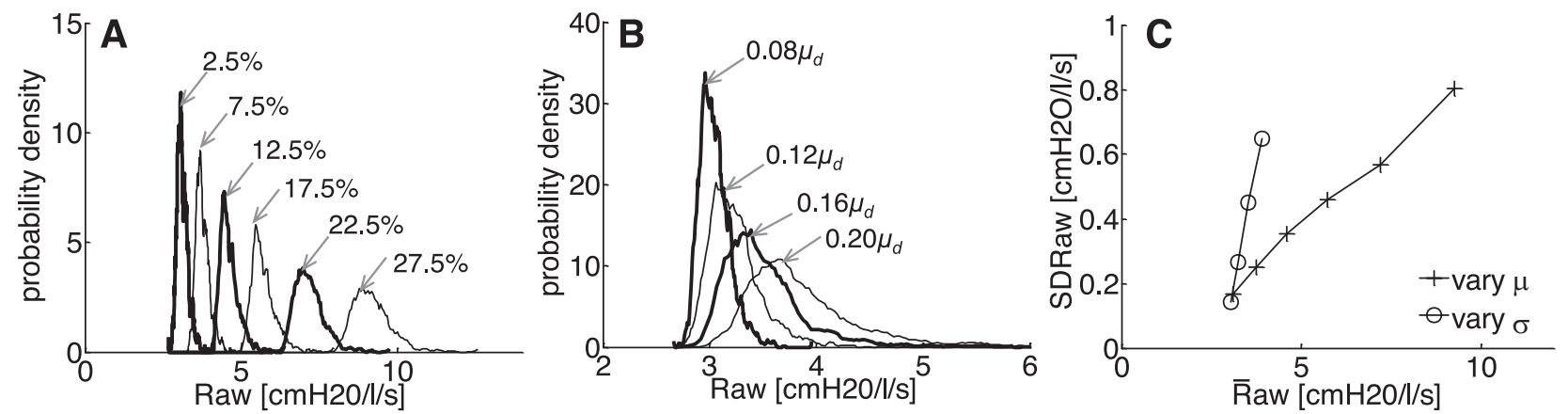

Fig. 2. Probability distributions of Raw for increasing $(A)$ mean diameter, while fixing the variability in diameter constant, and $(B)$ the variability in diameter at a fixed mean $\left(\mu_{d}\right)$. C: comparison of the respective variation in Raw as described by the SD (SDRaw) vs. mean Raw (Raw) for these simulations.

when $\sigma_{d}$ was increased at a fixed average diameter, the slope of SDRaw vs. $\bar{R}$ aw was greatest (Fig. 2C).

Imposing gas distribution abnormalities or ventilation defects. By closing different airways within each chosen generation-simulating regional gas distribution differences or ventilation defects - we created multiple simulations for single defects of different sizes within a generation (i.e., occluding four different airways at the third generation) and across generations (generations four to two). We observed that $\bar{R} a w$ increased as expected and was dependent on defect size, with larger defects causing a larger change in $\bar{R}$ aw (Fig. 3). The addition of ventilation defects resulted in an increase in SDRaw; however, this effect was modest compared with the effect of increasing $\bar{R} a w$, particularly when compared with the model with increasing diameter variation alone (Fig. 3).

Coherent variation in airway diameter. When the amount of coherency in airway variation between connected airways was increased from 0 to $100 \%$ with $10 \%$ intervals, the SDRaw increased substantially, and $\bar{R} a w$ remained largely unchanged, as shown in Fig. 3. Up until $\varphi$ began to exceed 0.6, there was little effect on SDRaw, but the effect on SDRaw increased greatly for larger $\varphi$. The common crossing point in Figs. 2C and 3 is the baseline condition, common to all simulations, with incoherent diameter fluctuations $\sigma_{d}$ at $10 \%$ of the diameter mean and without defects.

Combining with airway diameter narrowing. The above effects on the airway model were also tested at different airway diameters decreasing from FRC (Fig. 4A). Over the same range

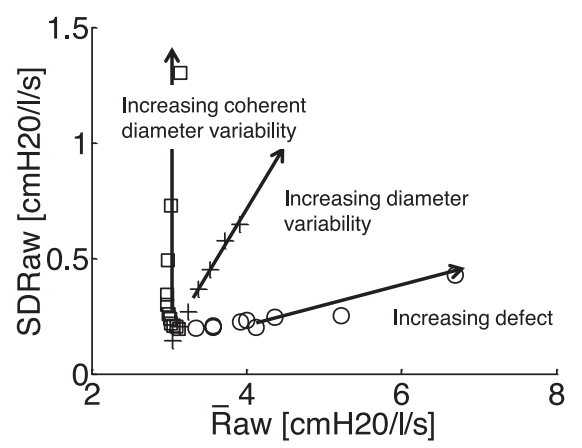

Fig. 3. This figure shows the dependence of SDRaw on $\bar{R}$ aw for increasing coherent variation, increasing diameter variability, and increasing defect size. Figure shows increasing airway diameter SD from $8 \%$ to $20 \%$ in $2 \%$ steps $(+)$, increasing airway variation coherency from $0 \%$ (incoherent) to $100 \%$ in $10 \%$ steps $(\square)$, and increasing the ventilation defect size from 4th generation airway to the 2 nd generation $(\bigcirc)$. in average airway diameters, a larger $\sigma_{d}$ led to a shift to both higher Raw and SDRaw, but SDRaw was more strongly affected, (Fig. $4 B$ ) as indicated by the higher SDRaw vs. $\bar{R} a w$ slope $\left(\right.$ slope $\left._{R}\right)$, which increased approximately twofold (Fig. 4F).

We also tested how the coherency of variation of the airway diameter at different constrictions would affect the probability distribution of $\bar{R} a w$ by comparing two extremes: the fully incoherent, where airways vary with no relationships to each other, and the coherent extreme, where all of the airways vary in unison. Comparing the results of these two extremes of incoherent and coherent variation, we found that the slope $_{R}$ was greater for a coherently varying airway tree by a factor of six, as can be seen when comparing Fig. $4 A$ with $C$ and $F$, respectively. While airways that vary totally in unison are inconceivable if driven actively by the smooth muscle, it is a reasonable approximation to breathing. Furthermore, the $\varphi=1$ case is the simplification of the multibranch lung to a single airway, and the case matching our single airway model provides further validation of the model (Fig. $4 E$ ).

The effects of adding a large defect can be seen in Fig. 4D, via obstruction in a second generation airway. The defect causes both the $\bar{R}$ aw and SDRaw to increase and maintains a nearly constant slope $_{R}$.

FOT data from patients. Data collected from asthmatic and normal patients by Que et al. (22) were analyzed by comparing the average histograms from each group, which were significantly different, as reported previously. Average Rrs from the asthmatic patients (Fig. $4 H$ ) were right shifted, with a more broad distribution and increases in SDRrs compared with normal patients (Fig. 4G).

\section{DISCUSSION}

Here, we used a statistical distribution approach to explore the sensitivity of $\bar{R} a w$ to several factors that can influence Raw levels and its variation, which may be presented in a multibranch airway tree, including dependence on diameter mean and variation, regional coherency of variation, and the presence of ventilation defects. We demonstrated that Gaussian variation in airway diameter could produce distributions in Raw, similar to that reported for Rrs in vivo. The presence of large ventilation defects and any decrease in average airway diameter increased both $\bar{R} a w$ as well as its variation and predicted a robust relationship between SDRaw and Raw, which mimicked the relationship between SDRrs and Rrs in vivo. The most potent influence on SDRaw was coherency in diameter 
A
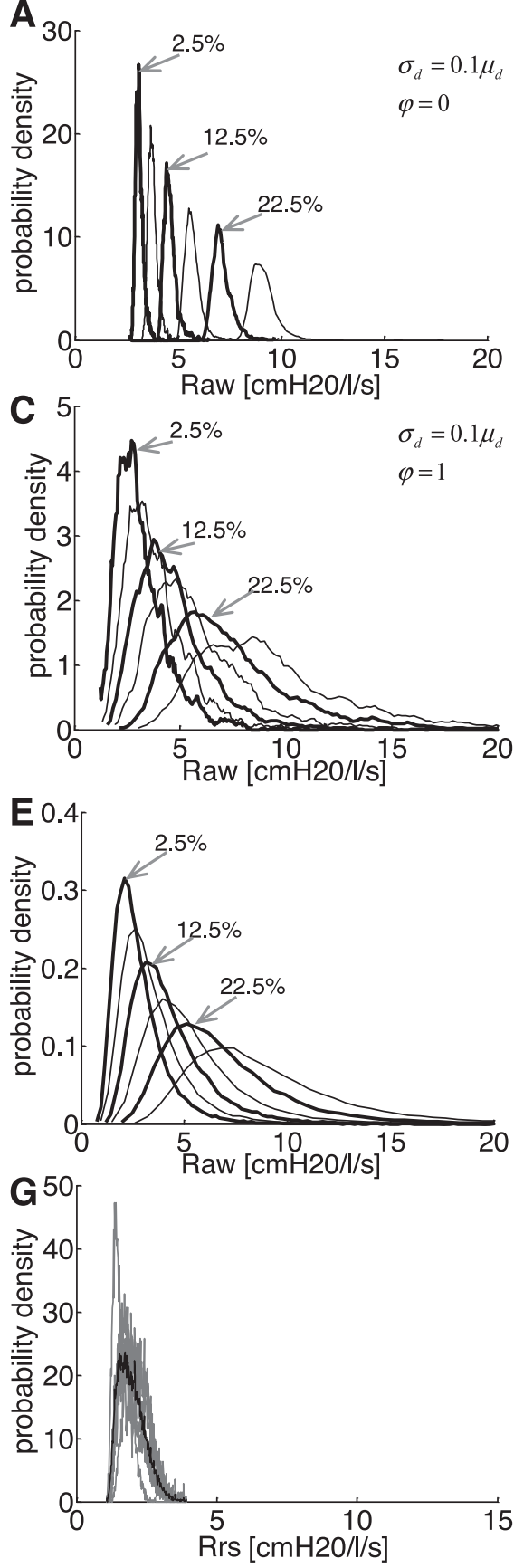

B
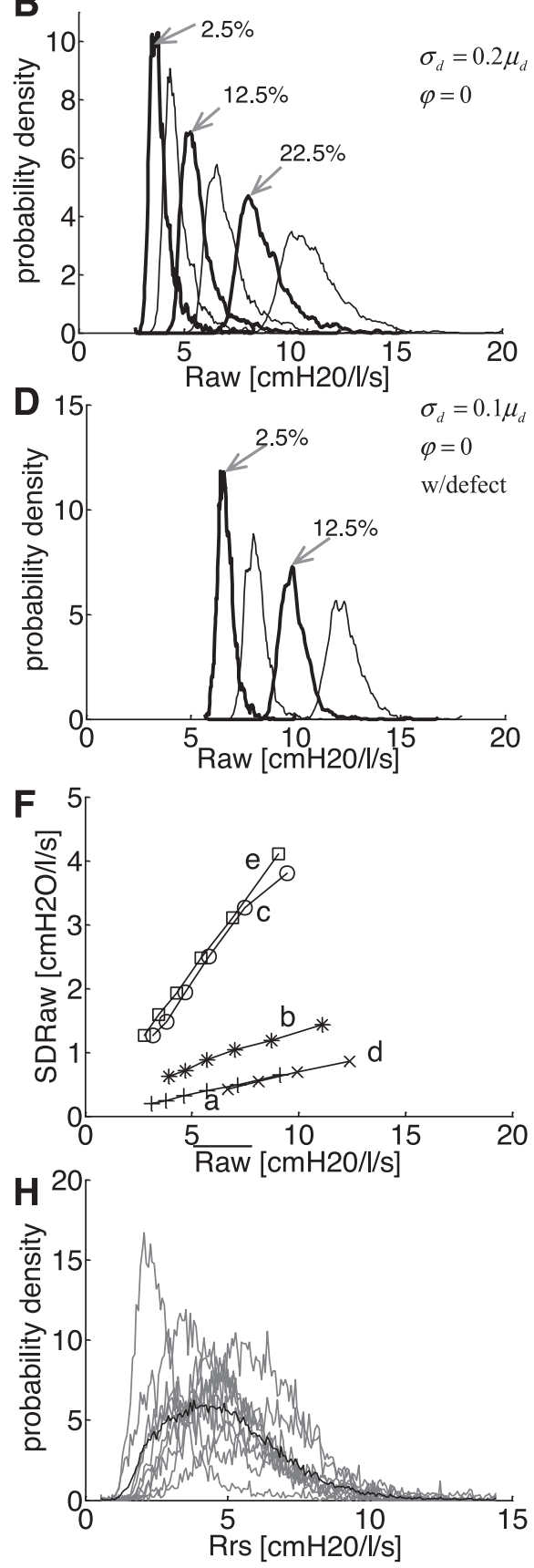

Fig. 4. Resistance histograms at various levels of mean airway constriction and different model effects $(B-E) . A$ : histograms are generated with the initial conditions of airway diameter $\left(\sigma_{d}\right)=0.1 \mu_{d}$, and $\varphi=0$, with no defects. $B: \sigma_{d}$ is doubled; $C$ : the coherency is set at its maximum of $\varphi=1$; and $D$ : a large defect is added at the 2nd generation airway. The total coherent scenario $(C)$ is very similar to the single airway model $(E) . F$ : the dependence of SDRaw on Raw for the different simulations. These simulated distributions can be compared with forced oscillation technique respiratory system resistance distributions from Que et al. (22), comparing normal subjects $(G)$ with asthma subjects $(H)$. Gray lines are the distribution from an individual, and bold lines indicate the mean for the group. variation between parent and daughter airways, but this was required to match FOT Rrs distributions.

Variation in lung function is a well-established feature of asthma over both long and short time scales. At long time scales, variation in peak expired flow is higher in asthma and is thought to arise from complex interactions between fluctuations in environmental stimuli and inflammatory status, amongst other factors (25). Interestingly, the variation in peak expired flow has been shown to exhibit temporal correlations that can be used to predict the likelihood of future declines in lung function, as measured by $\mathrm{FEV}_{1}(10)$. Variation at a shorter time scale is observed as fluctuations in airway impedance or resistance, which are increased in asthma, and the fluctuations are thought to arise from changes in the activity of the airway smooth muscle. While variation at long and short time scales both involves changes in airway function and thus diameter, it is not clear if it shares underlying mechanisms, although at both time scales it is increased in asthma. In our model, the distributions of airway diameter and thus the $\bar{R} a w$ distributions are independent of any time scale and could be used to examine either behavior. Here, we used the model to investigate variation over short time scales in frequency distributions of respiratory impedance, for which there is ample available data.

We showed that increasing the variation of airway diameters throughout the airway tree not only increased SDRaw but also $\bar{R} a w$. We have shown that the importance of variation in narrowing and coherency in variation at an anatomical level can alter the dependence between SDRaw and $\bar{R} a w$, whereas 
the effect of ventilation defects plays a negligible role. While we demonstrated several factors arising from the multibranching airway tree, which influence this dependence, the single airway model showed that this can arise solely from the transformation of the random diameter distribution via the inverse fourth-power law dependence of resistance on diameter $\bar{R} a w \propto 1 / d^{4}$. The strongly nonlinear geometric dependence of $\bar{R} a w$ on airway diameter strongly skews the original diameter distribution, producing long tails to high $\bar{R} a w$ values. When the diameter distribution of a single airway is widened through increasing $\sigma_{d}$, average Raw must increase, as the effects on $\bar{R} a w$ with decreasing diameter are much greater than with increasing diameter. Similarly, in a multibranch lung, airways that narrowed had a stronger effect on total lung resistance than those that dilate and would similarly skew the distribution, increasing both $\bar{R} a w$ and SDRaw. This was true even while the $\mathrm{SD}$ of airway diameter $\sigma_{d}$ was increased, and mean diameter $\mu_{d}$ was constant, increasing SDRaw and modestly increasing Raw (Fig. 2C). We found, importantly, that SDRaw was consistently dependent on $\bar{R} a w$. This occurred whether $\sigma_{d}$ was increased with no change in $\mu_{d}$ or when $\mu_{d}$ was decreased with no change in $\sigma_{d}$, although when $\sigma_{d}$ was unchanged, the slope was lower as expected (Fig. 2C). Similarly, when $\sigma_{d}$ was increased in proportion to $\mu_{d}$, such that they were increased together, keeping the slope $_{d}$ of airway diameters constant, SDRaw and Raw also increased linearly (Fig. 3). There exists an analytical solution for this particular case in the single airway model, helping to validate the model presented (17). The fact that a linear relationship is predicted in each case arises from the choice of variable, which was independently varied in each case, and does not mean that a linear relationship between SDRaw and $\bar{R} a w$ is inevitable, as changing both $\mu_{d}$ and $\sigma_{d}$ arbitrarily can produce any point bound by the top and bottom curves (see Fig. 2). However, the linear relationship well matches baseline Rrs and SDRrs data across subjects in asthma and health and for some subjects, pre- and postbronchodilator changes in Rrs and $S D R r$ within individual children with asthma (17). Thus it is possible that the conditions for linear dependence, may explain the reported linear dependence of SDRrs on Rrs in normal and asthmatic subjects, as we discuss further below. Furthermore, we explored several factors that may influence this dependence, which arise within a multibranch lung.

Regional coherent variation. In obstructive disease, the lungs tend to have regions that are poorly ventilated, often described as ventilation defects, observed using hyperpolarized ${ }^{3} \mathrm{He}$ MRI, PET, or CT, whereas other regions can be hyperventilated, thought to arise as the lung compensates to maintain an equal tidal volume. One mechanism thought to explain the development of regions of poor ventilation or "patchy" ventilation in asthma is the mechanism of bistability of airway diameters in the lung. This bistability intrinsically arises from the softening and dilating behavior of airway smooth muscle exposed to oscillations in loading, such as breathing, in combination with parenchymal tethering and bifurcating airways, as developed by Anafi and Wilson (1) in their model of a single terminal unit embedded in parenchyma and developed further in a multibranch lung model by Winkler and coworkers (31, 32 ), who demonstrated that the formation of regions of poor and well-ventilated regions in the lung grew as airway narrowing migrated serially up and down connected airways. This occurred as fluctuations afforded airways by parenchymal tethering were decreased by a local loss of ventilation. With less ventilation to a particular region of the lung, oscillations that act to relax airway smooth muscle decrease, allowing local spreading of airway narrowing, providing one explanation for why airway diameter variation could behave dependently and coherently. Of course, more directly, parent and daughter airways are mechanically connected and share local fluctuations in parenchymal tethering and thus would be expected to change diameters somewhat coherently. Here, we simulated the effects of this interdependence of airway diameter up and down the airway tree by using a coherency parameter $\varphi$, which controlled the similarity in dilation or contraction between parent airways and their daughters. We found that increasing coherency while the distribution of variation amongst airway diameters was unchanged led to patchiness in airway narrowing but also led to an increase in SDRaw with almost no change in $\bar{R} a w$. This was readily apparent with large $\varphi(>0.6$; Fig. 3). The patchiness in airway narrowing that we observed is reminiscent of the patchy ventilation reported by Winkler and coworkers $(31,32)$ in their models and subjects (see Supplemental Video). That coherency of airway diameter variation had perhaps the most potent effect on SDRaw relative to $\bar{R} a w$ implies that any mechanism leading to interdependence and regional airway narrowing tends to increase the slope of the SDRaw vs. $\bar{R}$ aw relationship, compared with simply random fluctuations in airway diameter within the airway tree.

As described above, SDRrs is proportional to Rrs across subjects with asthma and healthy controls, and in fact, all subjects appear to fall on a single relationship. Diba et al. (9) reported that SDRrs was highly correlated with Rrs with a regression coefficient of $r=0.81$ from 102 nonasthmatics, and $r=0.91$ from 38 asthmatics with apparently the same dependence, with a slope of 0.35 estimated from their data. Que et al. (22) also reported SDRrs and Rrs data from asthma and control subjects, and when we compute the slope here, it well matches the data of Diba et al. (9) with a slope of 0.31 , similar $y$-intercept, and $r=0.67$ (Fig. $5 A$ ). Indeed, the fact that two studies reporting SDRrs and Rrs from two different countries can have nearly the same slope $_{R}$ is noteworthy, given that the range in Rrs amongst subjects was from 1 to $7 \mathrm{cmH}_{2} \mathrm{O} \cdot 1^{-1} \cdot \mathrm{s}^{-1}$ and that asthmatic subjects in Que et al. (22) had a much higher resistance of $4.90(\mathrm{SD} 1.06) \mathrm{cmH}_{2} \mathrm{O} \cdot 1^{-1} \cdot \mathrm{s}^{-1}$ compared with the controls with Rrs of 1.80 (SD 1.06) $\mathrm{cmH}_{2} \mathrm{O} \cdot \mathrm{l}^{-1} \cdot \mathrm{s}^{-1}$. In both studies, subjects with asthma had higher Rrs but also, higher SDRrs in proportion, such that the presence of asthma is represented by a shift up the line rather than any change above or below the line. This is surprising, as it likely limits the number of mechanisms for how fluctuations in airway diameter may lead to fluctuations in Rrs. For example, explaining the increased SDRrs in asthma via increased coherency of ventilation alone is not likely, as this should lead to greater changes in SDRrs than Rrs.

As an exercise to compare our model with these measured data, we generated results, with a similar slope at $\varphi=0.88$ and $\sigma_{d}$ as a constant fraction of the mean diameter at $0.11 \mu_{d}$ (Fig. $5 A$ ). Whereas other parameter choices in models could nearly match the data, this model was perhaps the most reasonable compared with constant $\mu_{d}$ or $\sigma_{d}$ models, which are unlikely. Although Fig. 5A shows the SDRrs vs. Rrs relationship from Diba et al. (9), together with this multibranch model prediction, 

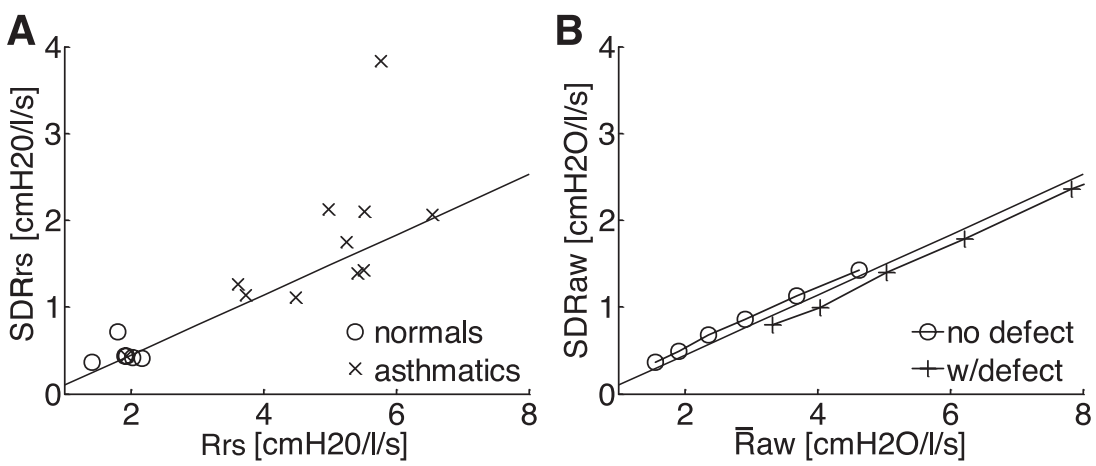

Fig. 5. A: comparing the slope of SDRaw with $\bar{R} a w$ from the model predictions with the estimated average SDRrs vs. Rrs slope value in patients measured from Diba et al. (9) and from Que et al. (22). $B$ : introducing a large defect into lungs with different levels of constriction ( $\xi$ increasing from $2.5 \%$, as described in METHODS) increased both Raw and SDRaw, as seen in the rightward and upward shift but along the same slope.

we do not imply that these parameters correspond precisely to the actual variation of airway diameters within the lung. One reason is that we limited the contribution to variation in $\bar{R} a w$ to arise solely from airway diameter fluctuations, whereas other factors will contribute to the total airway variation in Raw in a recording, such as breathing and transducer noise. However, the fact that tuning a simple coherency parameter and using a fixed slope $_{d}$ model predicts the same observed relationship as in FOT measurements between SDRaw and $\bar{R} a w$ is remarkable.

Defects. As mentioned, ventilation defects have been identified using hyperpolarized MRI and other techniques. They are commonly defined in MRI as a focal signal void within a region of the lung. Ventilation abnormalities are hallmark findings in obstructive airway-related lung diseases, such as asthma $(6,16)$ and chronic obstructive pulmonary disease (COPD) $(20,21)$, and can vary in volume and number, and importantly, these are absent in healthy, young never-smokers. We found that adding a large defect increased both $\bar{R} a w$ and $S D R a w$ but maintained a similar slope $e_{R}$ (Fig. $5 B$ ). The increase in $\bar{R} a w$ from a defect was expected, due to the loss in parallel Raw pathways, but the reason for the slight increase in SDRaw is less clear. A plausible mechanism is that because a defect removes some airways from contributing to $\mathrm{Raw}$, it thus also removes the ability of those airways to compensate for other airways in the unaffected portion of the lung. That is, the number of available configurations or states for the set of airway diameters within the lung was reduced, leading to an increased sensitivity of SDRaw to airway diameter variation. Indeed, with no defects, we would expect the overall variation in SDRaw to be its lowest, as there are a maximum number of airways varying, able to compensate for changes in each other. This predicts that stable defects, consistent with persistent defects observed in asthma (24), may contribute to increased variation in Raw, and larger or more numerous defects have a greater effect. However, while the increase in SDRaw could be reversed if either hyperventilation in nondefective regions or an increase in FRC were sufficient to increase mean airway diameter the increase in SDRaw from an imposed defect is consistent with SDRrs changes in Rrs observed with asthma (9, 17, 22).

Limitations. This model examined the effects of varying airway diameters on $\bar{R} a w$ and SDRaw, including some multibranch factors, such as coherency and ventilation defects. We did not seek to predict the temporal behavior of $\bar{R} a w$ here, concentrating only on parameters of the amplitude distribution, specifically $\bar{R} a w$ and its variation computed by SDRaw. Thus this model explicitly excluded temporal features of airway narrowing and does not track changes in $\bar{R} a w$ from excitationcontraction and load-dependent airway narrowing, as well as viscoelastic behavior or redistribution of ventilation via the pendelluft effect (19). The model was instead an examination of the potential effects on the statistical distribution of $\bar{R} a w$ of heterogeneity in airway narrowing, including changes in diameter fluctuation distributions and the presence of large-scale ventilation defects. This resulted in a computationally feasible examination of $\bar{R} a w$ and its distribution properties. However, it is possible that temporal dynamics may have an effect on the shapes of the distribution function for $\bar{R} a w$ and could thus alter the particular slopes that we determined between SDRaw and $\bar{R} a w$, for example. However, it is unlikely that temporal dynamics would greatly affect the roles of the mean airway diameter, SD of airway diameter, coherency, or presence of defects. Furthermore, temporal dynamics were apparently not needed to predict the established distribution profile for Rrs or reported correlation of SDRrs on Rrs.

As we did not have temporal dynamics, we also did not have breathing explicitly included in our model. However, if breathing is considered to be a homogenous effect, increasing and decreasing airway diameters, this condition is like that of the $100 \%$ coherently varying lung with airways varying in concert, for which we also explored, altering mean airway diameter and its SD. Other contributions to SDRaw obtained in vivo could include artifactual glottis closures, intermittent leaks, or events, such as coughs or swallows. Whereas obvious artifacts were removed by Diba et al. (9) and Que et al. (22), it is possible that some artifacts were undetected, contributing to increased SDRaw than would be predicted for a given parameter set in our model.

Similar to other models, we neglected any contribution to lung impedance from gas compression or airway elastance, as lung elastance is dominated by the highly compliant lung tissue $(15,16)$. Elastance was then assigned to the terminal airspaces and was essential, as these can contribute to determining Raw in a lung with time-constant heterogeneity, as first demonstrated by Otis (19). However, heterogeneity in airway compliances would likely somewhat broaden the distribution of Raw.

Physiological interpretation of constant slope $\mathrm{R}_{\mathrm{R}}$. Why SDRrs is proportional to Rrs, giving nearly a constant slope $_{R}$ of $\sim 0.35$ across all subjects, with or without asthma, is an interesting question. This implies that factors with a strong influence on the slope of SDRrs to Rrs are unlikely to vary substantially across individuals. Since coherency of ventilation had a potent effect in our model, whatever coherency in diameter motions 
that may be present in vivo between daughter and parent airways is unlikely to change much amongst individuals. Yet, why the ratio of SDRrs to Rrs should be so apparently consistent amongst individuals and be $\sim 0.35$ is unclear. We have shown in our model that changes in mean airway diameter, such as would occur with lung growth, airway constriction, dilation, or body size, do not affect the slope $_{R}$, and the effect can arise from a lung modeled as a single airway, which suggests that a dominant mechanism is from the inverse fourthpower law dependence of Raw on the diameter and thus is geometrical in origin, as suggested by Lall et al. (17).

The single airway or branching tree model did not include other sources of resistance, such as turbulent or transitional flow, which would occur in the glottis and upper airways. This could be better described using Wormesley flow rather than Poiseuille flow, but this is complicated, especially if interactions with breathing are considered. Louis and Isabey (18) indicated that this begins to affect Rrs, estimated by FOT, in humans at $7 \mathrm{~Hz}$ for breathing $>0.5 \mathrm{l} / \mathrm{s}$, which indicates that this may be a small factor, and we may underestimate lung resistance and could affect the slope $_{R}$ in our model. Either transitional or turbulent flow does not have any appreciable effect, as the model could produce similar linear SDRaw vs. $\bar{R} a w$ to that in vivo, or other factors may contribute to the fixed slope $_{R}$ in vivo. However, deviations from this relationship in vivo have been reported with bronchodilator administration. Lall et al. (17) found that changes in SDRrs with bronchodilator exceeded decreases in Rrs in a subset of children with asthma, and SDRrs and Rrs were not proportional in this group (17). This means that in some children, the additional variation in Rrs at baseline might have been attributable to additional abnormal fluctuations in airway diameter, possibly reflective of asthma control (11). If a consistent slope $_{R}$ arises largely from geometrical factors, then it is possible that at extreme lung volumes, slope $_{R}$ may be altered, but this has not been examined, and there was little change in the proportionality of SDRaw to Rrs with upright and supine positions (9).

We observed that a large defect could substantially increase $\bar{R} a w$ and SDRaw, shifting the values rightward and upward but with no change in the slope of SDRaw to $\bar{R} a w$ (Fig. $5 B$ ). The fact that adding a defect led to an increase in $\bar{R} a w$ is intuitive, but that it has a similar effect on its variation is not. This prediction is potentially useful, as defects are known to increase in both volume and number in asthmatic $(5,7)$ as well as $\operatorname{COPD}(20,21)$ patients and are thought to be largely persistent over time scales of hours to weeks (25).

In summary, we observed several conditions that predicted a linear relationship between $\bar{R} a w$ and SDRaw, similar to established data. Likely, SDRaw and $\bar{R} a w$ together depend on airway diameter as described above, and both scale with homogeneous changes in airway dimensions, on average, as might occur with bronchoconstrictors and bronchodilators. The model predicted that airways must exhibit some localized coherency in airway diameter changes to exhibit regional ventilation heterogeneity and to approximate the reported slope of SDRrs to Rrs. These findings establish possible mechanisms for the relationship between SDRrs on Rrs. An increase in the SDRaw with an increase in defect size was also predicted, possibly representing an emergent phenomenon of the model arising from a reduced number of available configurations within the airway tree and thus increasing sensitivity of $\bar{R} a w$ to fluctuations amongst airway diameters. Thus SDRaw can be altered by several possible mechanisms independent of $\bar{R} a w$, yet the many mechanisms that act to constrain SDRaw and $\bar{R} a w$ support that this is likely a robust relationship that cannot be easily altered.

\section{ACKNOWLEDGMENTS}

The authors thank M. Tawhai for the generous provision of the airway dimension data.

\section{GRANTS}

This work was supported by a Natural Sciences and Engineering Research Council of Canada Strategic Operating Grant in partnership with an Atlantic Innovation Fund award from the Atlantic Canada Opportunities Agency. D. Leary is supported by the Canadian Thoracic Society studentship.

\section{DISCLOSURES}

No conflicts of interest, financial or otherwise, are declared by the author(s).

\section{AUTHOR CONTRIBUTIONS}

Author contributions: D.L., S.A.B., and G.N.M. conception and design of research; D.L. performed experiements; D.L. analyzed data; D.L., S.A.B., G.P., and G.N.M. interpreted results of experiments; D.L. prepared figures; D.L. drafted manuscript; D.L., G.P., and G.N.M. edited and revised manuscript; D.L., S.A.B., G.P., and G.N.M. apprvoed final version of manuscript.

\section{REFERENCES}

1. Anafi RC, Wilson TA. Airway stability and heterogeneity in the constricted lung. J Appl Physiol 91: 1185-1192, 2001.

2. Black LD, Dellaca R, Jung K, Atileh H, Israel E, Ingenito EP, Lutchen KR. Tracking variations in airway caliber by using total respiratory vs. airway resistance in healthy and asthmatic subjects. J Appl Physiol 95: 511-518, 2003.

3. Brown RH, Kaczka DW, Fallano K, Chen S, Mitzner W. Temporal variability in the responses of individual canine airways to methacholine. J Appl Physiol 104: 1381-1386, 2008.

4. Campana L, Kenyon J, Zhalehdoust-Sani S, Tzeng YS, Sun Y, Albert M, Lutchen KR. Probing airway conditions governing ventilation defects in asthma via hyperpolarized MRI image functional modeling. J Appl Physiol 106: 1293-1300, 2009.

5. de Lange EE, Altes TA, Patrie JT, Battiston JJ, Juersivich AP, Mugler JP III, Platts-Mills TA. Changes in regional airflow obstruction over time in the lungs of patients with asthma: evaluation with $3 \mathrm{He} \mathrm{MR}$ imaging. Radiology 250: 567-575, 2009.

6. de Lange EE, Altes TA, Patrie JT, Parmar J, Brookeman JR, Mugler JP 3rd, Platts-Mills TA. The variability of regional airflow obstruction within the lungs of patients with asthma: assessment with hyperpolarized helium-3 magnetic resonance imaging. J Allergy Clin Immunol 119: 1072-1078, 2007.

7. de Lange EE, Mugler JP 3rd, Brookeman JR, Knight-Scott J, Truwit JD, Teates CD, Daniel TM, Bogorad PL, Cates GD. Lung air spaces: $\mathrm{MR}$ imaging evaluation with hyperpolarized $3 \mathrm{He}$ gas. Radiology 210 : 851-857, 1999.

8. Delacourt C, Lorino H, Herve-Guillot M, Reinert P, Harf A, Housset B. Use of the forced oscillation technique to assess airway obstruction and reversibility in children. Am J Respir Crit Care Med 161: 730-736, 2000.

9. Diba C, Salome CM, Reddel HK, Thorpe CW, Toelle B, King GG. Short term variability of airway calibre - a marker of asthma? J Appl Physiol 2007.

10. Frey U, Brodbeck T, Majumdar A, Taylor DR, Town GI, Silverman M, Suki B. Risk of severe asthma episodes predicted from fluctuation analysis of airway function. Nature 438: 667-670, 2005.

11. Frey U, Maksym GN, Suki B. Temporal complexity in clinical manifestations of lung disease. J Appl Physiol 2011.

12. Horsfield K, Cumming G. Morphology of the bronchial tree in man. $J$ Appl Physiol 24: 373-383, 1968.

13. Jensen A, Atileh H, Suki B, Ingenito EP, Lutchen KR. Selected contribution: airway caliber in healthy and asthmatic subjects: effects of bronchial challenge and deep inspirations. J Appl Physiol 91: 506-515, 2001 . 
14. Kaczka DW, Brown RH, Mitzner W. Assessment of heterogeneous airway constriction in dogs: a structure-function analysis. J Appl Physiol 106: 520-530, 2009.

15. Kaczka DW, Ingenito EP, Israel E, Lutchen KR. Airway and lung tissue mechanics in asthma. Effects of albuterol. Am J Respir Crit Care Med 159: 169-178, 1999.

16. Kaczka DW, Ingenito EP, Suki B, Lutchen KR. Partitioning airway and lung tissue resistances in humans: effects of bronchoconstriction. J Appl Physiol 82: 1531-1541, 1997.

17. Lall CA, Cheng N, Hernandez P, Pianosi PT, Dali Z, Abouzied A, Maksym GN. Airway resistance variability and response to bronchodilator in children with asthma. Eur Respir J 30: 260-268, 2007.

18. Louis B, Isabey D. Interaction of oscillatory and steady turbulent flows in airway tubes during impedance measurement. J Appl Physiol 74: $116-$ $125,1993$.

19. Otis AB, McKerrow CB, Bartlett RA, Mead J, McIlroy MB, SelverStone NJ, Radford EP Jr. Mechanical factors in distribution of pulmonary ventilation. J Appl Physiol 8: 427-443, 1956.

20. Parraga G, Mathew L, Etemad-Rezai R, McCormack DG, Santyr GE. Hyperpolarized $3 \mathrm{He}$ magnetic resonance imaging of ventilation defects in healthy elderly volunteers: initial findings at 3.0 Tesla. Acad Radiol 15: 776-785, 2008.

21. Parraga G, Ouriadov A, Evans A, McKay S, Lam WW, Fenster A, Etemad-Rezai R, McCormack D, Santyr G. Hyperpolarized $3 \mathrm{He}$ ventilation defects and apparent diffusion coefficients in chronic obstructive pulmonary disease: preliminary results at 3.0 Tesla. Invest Radiol 42: 384-391, 2007.

22. Que CL, Kenyon CM, Olivenstein R, Macklem PT, Maksym GN. Homeokinesis and short-term variability of human airway caliber. $J$ Appl Physiol 91: 1131-1141, 2001.

23. Salerno M, Altes TA, Mugler JP 3rd, Nakatsu M, Hatabu H, de Lange EE. Hyperpolarized noble gas MR imaging of the lung: potential clinical applications. Eur J Radiol 40: 33-44, 2001.
24. Samee S, Altes T, Powers P, de Lange EE, Knight-Scott J, Rakes G, Mugler JP III, Ciambotti JM, Alford BA, Brookeman JR, PlattsMills TA. Imaging the lungs in asthmatic patients by using hyperpolarized helium-3 magnetic resonance: assessment of response to methacholine and exercise challenge. J Allergy Clin Immunol 111: 1205-1211, 2003.

25. Suki B, Frey U. Temporal dynamics of recurrent airway symptoms and cellular random walk. J Appl Physiol 95: 2122-2127, 2003.

26. Tawhai MH, Hunter P, Tschirren J, Reinhardt J, McLennan G, Hoffman EA. CT-based geometry analysis and finite element models of the human and ovine bronchial tree. J Appl Physiol 97: 2310-2321, 2004.

27. Tawhai MH, Nash MP, Lin CL, Hoffman EA. Supine and prone differences in regional lung density and pleural pressure gradients in the human lung with constant shape. J Appl Physiol 107: 912-920, 2009.

28. Tgavalekos NT, Musch G, Harris RS, Vidal Melo MF, Winkler T, Schroeder T, Callahan R, Lutchen KR, Venegas JG. Relationship between airway narrowing, patchy ventilation and lung mechanics in asthmatics. Eur Respir J 29: 1174-1181, 2007.

29. Tgavalekos NT, Venegas JG, Suki B, Lutchen KR. Relation between structure, function, and imaging in a three-dimensional model of the lung. Ann Biomed Eng 31: 363-373, 2003.

30. Tzeng YS, Lutchen K, Albert M. The difference in ventilation heterogeneity between asthmatic and healthy subjects quantified using hyperpolarized 3He MRI. J Appl Physiol 106: 813-822, 2009.

31. Venegas JG, Winkler T, Musch G, Vidal Melo MF, Layfield D, Tgavalekos N, Fischman AJ, Callahan RJ, Bellani G, Harris RS. Self-organized patchiness in asthma as a prelude to catastrophic shifts. Nature 434: 777-782, 2005.

32. Winkler T, Venegas JG. Complex airway behavior and paradoxical responses to bronchoprovocation. J Appl Physiol 103: 655-663, 2007. 